\title{
Operators and spaces associated to matrices with grades and their decompositions II
}

\author{
Radim Belohlavek and Jan Konecny \\ Dept. Computer Science \\ Palacky University, Olomouc \\ 17. listopadu 12, CZ-77146 Olomouc \\ Czech Republic \\ email: \{radim.belohlavek,jan.konecny\}@upol.cz
}

\begin{abstract}
The paper is a continuation of our previous paper on operators and spaces associated to matrices whose degrees are elements from a residuated lattice. The motivation for this study is to develop a calculus for such matrices which can be used in situations such as matrix decompositions. In this paper, we focus on row and column spaces, left and right ideals of matrices, and Green's relations. We prove basic results concerning these notions, show links to known structures, and put a new perspective on results known from Boolean matrices and their generalizations.
\end{abstract}

\section{Introduction}

In this paper, we continue the study, started in [6], of operators and spaces associated to matrices with entries from residuated lattices. Our main motivation is recent results on the role of closure and interior structures, most importantly concept lattices, for decompositions of such matrices and factor analysis of data described by such matrices $[7,8,10,9]$. In [6], we presented results regarding the operators and spaces associated to products of matrices and established some links to traditionally studied concepts from Boolean matrices. We showed that some of the concepts of Boolean matrix theory, such as the concept of a row space, can be naturally defined in terms of closure structures known from the theory of ordered sets and, in particular, in terms of structures known formal concept analysis of binary data [11]. Furthermore, we suggested that the concepts of Boolean matrix theory can be extended to matrices with entries from residuated lattices by utilizing the existing and possibly developing new notions and results of formal concept analysis of data with fuzzy attributes. A study of these topic is the primary purpose of the present paper.

The paper is organized as follows. Preliminaries are provided in Section 2. Section 3 presents the results, namely the results on rows and column spaces, and the results on Green's relations for matrices with entries from residuated lattices. Section 4 presents conclusions and topics for future research. 


\section{Preliminaries: Matrices, Compositions, Decompositions, Concept Lattices}

This section provides preliminaries, mainly from [6-10].

Matrices We deal with matrices whose degrees are elements from residuated lattices. In particular, we deal with (de)compositions $I=A * B$ which involve an $n \times m$ matrix $I$, an $n \times k$ matrix $A$, and a $k \times m$ matrix $B$. The entries of matrices $I, A, B$, are elements of a residuated lattice (see below); for the composition operator $*$, we use three particular products (explained later in this section).

Recall that a (complete) residuated lattice $[3,14,19]$ is a structure $\mathbf{L}=$ $\langle L, \wedge, \vee, \otimes, \rightarrow, 0,1\rangle$ such that

(i) $\langle L, \wedge, \vee, 0,1\rangle$ is a (complete) lattice, i.e. a partially ordered set in which arbitrary infima and suprema exist;

(ii) $\langle L, \otimes, 1\rangle$ is a commutative monoid, i.e. $\otimes$ is a binary operation which is commutative, associative, and $a \otimes 1=a$ for each $a \in L$;

(iii) $\otimes$ and $\rightarrow$ satisfy adjointness, i.e. $a \otimes b \leq c$ iff $a \leq b \rightarrow c$.

0 and 1 denote the least and greatest elements of $\mathbf{L}$. The partial order of $\mathbf{L}$ is denoted by $\leq$; infima and suprema in $\mathbf{L}$ by $\wedge$ and $\vee$. Throughout the paper, $\mathbf{L}$ denotes an arbitrary complete residuated lattice. Common examples of complete residuated lattices include those defined on a unit interval, i.e. $L=[0,1]$, or on a finite chain in a unit interval, e.g. $L=\left\{0, \frac{1}{n}, \ldots, \frac{n-1}{n}, 1\right\}$.

We assume that $I_{i j}, A_{i l}, B_{l j} \in L$. That is, all the matrix entries are elements of some complete residuated lattice $\mathbf{L}$. Elements $a \in L$ are also called grades (degrees). In particular $I_{i j}$ is interpreted as the degree to which object $i$ has attribute $j ; A_{i l}$ as the degree to which factor $l$ applies to object $i ; B_{l j}$ as the degree to which attribute $j$ is a manifestation (one of possibly several manifestations) of factor $l$.

Therefore, examples of matrices $I$ which are subject to our decomposition are

$$
\left(\begin{array}{lllllll}
1.0 & 1.0 & 0.0 & 0.0 & 0.6 & 0.4 \\
1.0 & 0.9 & 0.0 & 0.0 & 1.0 & 0.8 \\
1.0 & 1.0 & 0.0 & 1.0 & 0.0 & 0.0 \\
1.0 & 0.5 & 0.0 & 0.7 & 1.0 & 0.4
\end{array}\right) \text { or }\left(\begin{array}{lllll}
0 & 0 & 1 & 1 & 1 \\
0 & 0 & 1 & 1 & 0 \\
0 & 0 & 0 & 0 & 1 \\
0 & 1 & 1 & 1 & 0
\end{array}\right) \text {. }
$$

The second matrix demonstrates that binary matrices are a particular case of the matrices for $L=\{0,1\}$.

Remark 1. Residuated lattices are heavily used in fuzzy logic [3,14]. Operations $\otimes$ (multiplication) and $\rightarrow$ (residuum) play the role of a (truth function of) conjunction and implication, respectively. Examples of residuated lattices are well known. For instance, for $L=[0,1]$, we can use any left-continuous t-norm for $\otimes$, such as minimum, product, or Łukasewicz, and the corresponding residuum $\rightarrow$. 
Composition Operators We use three composition operators, $\circ, \triangleleft$, and $\triangleright$, and consider the corresponding decompositions $I=A \circ B, I=A \triangleleft B$, and $I=A \triangleright B$. The composition operators are defined by

$$
\begin{aligned}
& (A \circ B)_{i j}=\bigvee_{l=1}^{k} A_{i l} \otimes B_{l j}, \\
& (A \triangleleft B)_{i j}=\bigwedge_{l=1}^{k} A_{i l} \rightarrow B_{l j}, \\
& (A \triangleright B)_{i j}=\bigwedge_{l=1}^{k} B_{l j} \rightarrow A_{i l} .
\end{aligned}
$$

Note that these operators were extensively studied by Bandler and Kohout, see e.g. [17]. They have natural verbal descriptions. For instance, $(A \circ B)_{i j}$ is the truth degree of "there is factor $l$ such that $l$ applies to object $i$ and attribute $j$ is a manifestation of $l$ ". Note also that in the binary case, i.e. $L=\{0,1\}, A \circ B$ coincides with the well-known Boolean product of matrices.

Decomposition Problem Given an $n \times m$ matrix $I$ and a composition operator * (i.e., $\circ, \triangleleft$, or $\triangleright$ ), the decomposition problem consists in finding a decomposition $I=A * B$ of $I$ into an $n \times k$ matrix $A$ and an $k \times m$ matrix $B$ with the number $k$ (number of factors) as small as possible. The smallest $k$ is called the Schein rank of $I$ and is denoted by $\rho_{s}(I)$ (to make the type of product explicit, also by $\rho_{s \circ}(I), \rho_{s \triangleleft}(I)$, and $\left.\rho_{s \triangleright}(I)\right)$. Looking for decompositions $I=A * B$ can be seen as looking for factors in data described by $I$. That is, decomposing $I$ can be regarded as factor analysis in which the data as well as the operations used are different from the ordinary factor analysis [15].

Concept Lattices Associated to $I$ Let $X=\{1,2, \ldots, n\}$ and $Y=\{1,2, \ldots, m\}$. Recall that $L^{U}$ denotes the set of all $L$-sets in $U$, i.e. all mappings from $U$ to $L$. Consider the following pairs of operators induced by matrix $I$. First, a pair $\left\langle{ }^{\uparrow}, \downarrow\right\rangle$ of operators ${ }^{\uparrow}: L^{X} \rightarrow L^{Y}$ and $\downarrow$ : $L^{Y} \rightarrow L^{X}$ is defined by

$$
\begin{aligned}
& C^{\uparrow}(j)=\bigwedge_{i=1}^{n}\left(C(i) \rightarrow I_{i j}\right), \\
& D^{\downarrow}(i)=\bigwedge_{j=1}^{m}\left(D(j) \rightarrow I_{i j}\right),
\end{aligned}
$$

for $j \in\{1, \ldots, m\}$ and $i \in\{1, \ldots, n\}$. Second, a pair $\langle\cap, \cup\rangle$ of operators $\cap$ : $L^{X} \rightarrow L^{Y}$ and ${ }^{\cup}: L^{Y} \rightarrow L^{X}$ is defined by

$$
\begin{aligned}
& C^{\cap}(j)=\bigvee_{i=1}^{n}\left(C(i) \otimes I_{i j}\right), \\
& D^{\cup}(i)=\bigwedge_{j=1}^{m}\left(I_{i j} \rightarrow D(j)\right),
\end{aligned}
$$

for $j \in\{1, \ldots, m\}$ and $i \in\{1, \ldots, n\}$. Third, a pair $\left\langle\wedge,{ }^{\vee}\right\rangle$ of operators ${ }^{\wedge}: L^{X} \rightarrow$ $L^{Y}$ and ${ }^{\vee}: L^{Y} \rightarrow L^{X}$ is defined by

$$
\begin{aligned}
C^{\wedge}(j) & =\bigwedge_{i=1}^{n}\left(I_{i j} \rightarrow C(i)\right), \\
D^{\vee}(i) & =\bigvee_{j=1}^{m}\left(D(j) \otimes I_{i j}\right),
\end{aligned}
$$

for $j \in\{1, \ldots, m\}$ and $i \in\{1, \ldots, n\} .\langle\uparrow, \downarrow\rangle$ forms an antitone Galois connection, $\langle\cap, \cup\rangle$ and $\langle\wedge, \vee\rangle$ each form an isotone Galois connection. To emphasize that the operators are induced by $I$, we also denote the operators by

$$
\left\langle{ }^{\uparrow},{ }^{{ }_{I}}\right\rangle,\left\langle{ }^{\cap_{I}},{ }^{\cup_{I}}\right\rangle \text {, and }\left\langle\wedge^{\wedge_{I}},{ }^{\vee_{I}}\right\rangle \text {. }
$$


Furthermore, denote the corresponding sets of fixpoints by $\mathcal{B}\left(X^{\uparrow}, Y^{\downarrow}, I\right), \mathcal{B}\left(X^{\cap}, Y^{\cup}, I\right)$, and $\mathcal{B}\left(X^{\wedge}, Y^{\vee}, I\right)$, i.e.

$$
\begin{aligned}
\mathcal{B}\left(X^{\uparrow}, Y^{\downarrow}, I\right) & =\left\{\langle C, D\rangle \mid C^{\uparrow}=D, D^{\downarrow}=C\right\}, \\
\mathcal{B}\left(X^{\cap}, Y^{\cup}, I\right) & =\left\{\langle C, D\rangle \mid C^{\cap}=D, D^{\cup}=C\right\}, \\
\mathcal{B}\left(X^{\wedge}, Y^{\vee}, I\right) & =\left\{\langle C, D\rangle \mid C^{\wedge}=D, D^{\vee}=C\right\} .
\end{aligned}
$$

The sets of fixpoints are complete lattices, called concept lattices associated to $I$, and their elements are called formal concepts. Note that these operators and their sets of fixpoints have extensively been studied, see e.g. [1,2,4,12,18]. Note that if $L=\{0,1\}, \mathcal{B}\left(X^{\uparrow}, Y^{\downarrow}, I\right)$ coincides with the ordinary concept lattice of the formal context consisting of $X, Y$, and the binary relation (represented by) $I$.

The concept lattices associated to $I$ play a fundamental role for decompositions of $I$. Namely, given a set

$$
\mathcal{F}=\left\{\left\langle C_{1}, D_{1}\right\rangle, \ldots,\left\langle C_{k}, D_{k}\right\rangle\right\}
$$

of $L$-sets $C_{l}$ and $D_{l}$ in $\{1, \ldots, n\}$ and $\{1, \ldots, m\}$, respectively, define $n \times k$ and $k \times m$ matrices $A_{\mathcal{F}}$ and $B_{\mathcal{F}}$ by

$$
\left(A_{\mathcal{F}}\right)_{i l}=\left(C_{l}\right)(i) \quad \text { and } \quad\left(B_{\mathcal{F}}\right)_{l j}=\left(D_{l}\right)(i) .
$$

This says: the $l$-th column of $A_{\mathcal{F}}$ is the transpose of the vector corresponding to $C_{l}$ and the $l$-th row of $B_{\mathcal{F}}$ is the vector corresponding to $D_{l}$. Then, we have:

Theorem 1 (universality, $[\mathbf{7}, \mathbf{1 0}])$. (०) For every I there exists $\mathcal{F} \subseteq \mathcal{B}\left(X^{\uparrow}, Y^{\downarrow}, I\right)$ such that $I=A_{\mathcal{F}} \circ B_{\mathcal{F}}$.

(১) For every I there exists $\mathcal{F} \subseteq \mathcal{B}\left(X^{\cap}, Y^{\cup}, I\right)$ such that $I=A_{\mathcal{F}} \triangleleft B_{\mathcal{F}}$.

( $\triangleright$ ) For every $I$ there exists $\mathcal{F} \subseteq \mathcal{B}\left(X^{\wedge}, Y^{\vee}, I\right)$ such that $I=A_{\mathcal{F}} \triangleright B_{\mathcal{F}}$.

Theorem 2 (optimality, $[\mathbf{7}, \mathbf{1 0}]$ ). (०) Let $I=A \circ B$ for $n \times k$ and $k \times m$ matrices $A$ and $B$. Then there exists $\mathcal{F} \subseteq \mathcal{B}\left(X^{\uparrow}, Y^{\downarrow}, I\right)$ with $|\mathcal{F}| \leq k$ such that for the $n \times|\mathcal{F}|$ and $|\mathcal{F}| \times m$ matrices $A_{\mathcal{F}}$ and $B_{\mathcal{F}}$ we have $I=A_{\mathcal{F}} \circ B_{\mathcal{F}}$.

$(\triangleleft)$ Let $I=A \triangleleft B$ for $n \times k$ and $k \times m$ matrices $A$ and $B$. Then there exists $\mathcal{F} \subseteq \mathcal{B}\left(X^{\cap}, Y^{\cup}, I\right)$ with $|\mathcal{F}| \leq k$ such that for the $n \times|\mathcal{F}|$ and $|\mathcal{F}| \times m$ matrices $A_{\mathcal{F}}$ and $B_{\mathcal{F}}$ we have $I=A_{\mathcal{F}} \triangleleft B_{\mathcal{F}}$.

( $\triangleright$ Let $I=A \triangleright B$ for $n \times k$ and $k \times m$ matrices $A$ and $B$. Then there exists $\mathcal{F} \subseteq \mathcal{B}\left(X^{\wedge}, Y^{\vee}, I\right)$ with $|\mathcal{F}| \leq k$ such that for the $n \times|\mathcal{F}|$ and $|\mathcal{F}| \times m$ matrices $A_{\mathcal{F}}$ and $B_{\mathcal{F}}$ we have $I=A_{\mathcal{F}} \triangleright B_{\mathcal{F}}$.

\section{Row and Column Spaces, and Green's relations}

\subsection{Row and Column Spaces}

In Boolean matrix theory [16], row and column spaces play a crucial role. In

$[6]$ we shortly presented a connection between the row and column spaces on 
one hand, and the concept lattices associated to matrices, on the other hand. In this section, we recall and further develop this connection and related notions for matrices with entries from residuated lattices. For convenience, we identify vectors and matrices with entries from residuated lattices with fuzzy sets and fuzzy relations. That is, a vector $\left\langle a_{1}, \ldots, a_{n}\right\rangle$ with entries $a_{i} \in L$ is sometimes viewed as a fuzzy set $C$ in an $n$-element universe set, say $\{1, \ldots, n\}$ such that $C(i)=a_{i}$ for $i=1, \ldots, n$, and the like.

By $L^{n}$ and $L^{n \times m}$, we denote the set of all vectors with $n$ components (i.e., $1 \times n$ matrices) and the set of all $n \times m$ matrices with entries from $L$ (or, the set of all fuzzy sets in an $n$-element universe and the set of all fuzzy relations between an $n$ - and $m$-element universes). Using the terminology of Boolean matrices, we define the following notions, generalizing the notions known from Boolean matrices.

Definition 1. $V \subseteq L^{n}$ is called an i-subspace (or just a subspace if there is no danger of confusion) if

- $V$ is closed under $\otimes$-multiplication, i.e. for every $a \in L$ and $C \in V, a \otimes C \in V$ (here, $a \otimes C$ is defined by $(a \otimes C)(i)=a \otimes C(i)$ for $i=1, \ldots, n)$;

- $V$ is closed under $\bigvee$-union, i.e. for $C_{j} \in V(j \in J)$ we have $\bigvee_{j \in J} C_{j} \in V$ (here, $\bigvee_{j \in J} C_{j}$ is defined by $\left.\left(\bigvee_{j \in J} C_{j}\right)(i)=\bigvee_{j \in J} C_{j}(i)\right)$.

$V \subseteq L^{n}$ is called a c-subspace (or just a subspace if there is no danger of confusion) if

- $V$ is closed under $\rightarrow-$ shift, i.e. for every $a \in L$ and $C \in V, a \rightarrow C \in V$ (here, $a \rightarrow C$ is defined by $(a \rightarrow C)(i)=a \rightarrow C(i)$ for $i=1, \ldots, n)$;

- $V$ is closed under $\bigwedge$-intersection, i.e. for $C_{j} \in V(j \in J)$ we have $\bigwedge_{j \in J} C_{j} \in$ $V$ (here, $\bigwedge_{j \in J} C_{j}$ is defined by $\left.\left(\bigwedge_{j \in J} C_{j}\right)(i)=\bigwedge_{j \in J} C_{j}(i)\right)$.

Remark 2. (1) If $V \in L^{n}$ are regarded as fuzzy sets, the concepts of an i-subspace and a c-subspace coincide with the concept of a fuzzy interior operator and a fuzzy closure operator as defined in $[2,5]$.

(2) For $L=\{0,1\}$ the concept of an i-subspace coincides with the concept of a subspace from the theory of Boolean matrices [16]. In fact, closedness under $\otimes$-multiplication is satisfied for free in the case of Boolean matrices. Note also that for Boolean matrices, $V$ forms a c-subspace iff $\bar{V}=\{\bar{C} \mid C \in V\}$ forms an isubspace (with $\bar{C}$ defined by $\bar{C}(i)=\overline{C(i)}$ where $\bar{a}=a \rightarrow 0$, i.e. $\overline{0}=1$ and $\overline{1}=0$ ), and vice versa. However, such a reducibility among the concepts of i-subspace and c-subspace is not available in general because in residuated lattices, the law of double negation (saying that $(a \rightarrow 0) \rightarrow 0=a$ ) does not hold.

Definition 2. The i-span (c-span) of $V \subseteq L^{n}$ is the intersection of all $i$ subspaces (c-subspaces) of $L^{n}$ that contain $V$. The row i-space (row c-space) of matrix $I \in L^{n \times m}$ is the $i$-span (c-span) of the set of all rows of $I$ (considered as vectors from $L^{n}$ ). The column i-space (column c-space) is defined analogously as the $i$-span (c-span) of the set of columns of $I$. The row $i$-space, row c-space, column $i$-space, and column c-space of matrix I is denoted by $R_{i}(I), R_{c}(I), C_{i}(I)$, $C_{c}(I)$. 
Remark 3. For $L=\{0,1\}$, the row i-space of $I$ coincides with the row space of $I$ as defined in Boolean matrix theory.

For the concept lattices $\mathcal{B}\left(X^{\uparrow}, Y^{\downarrow}, I\right)$, denote the corresponding sets of extents and intents by $\operatorname{Ext}\left(X^{\uparrow}, Y^{\downarrow}, I\right)$ and $\operatorname{Int}\left(X^{\uparrow}, Y^{\downarrow}, I\right)$. That is,

$$
\begin{aligned}
\operatorname{Ext}\left(X^{\uparrow}, Y^{\downarrow}, I\right) & =\left\{C \in L^{X} \mid\langle C, D\rangle \in \mathcal{B}\left(X^{\uparrow}, Y^{\downarrow}, I\right) \text { for some } D\right\}, \\
\operatorname{Int}\left(X^{\uparrow}, Y^{\downarrow}, I\right) & =\left\{D \in L^{Y} \mid\langle C, D\rangle \in \mathcal{B}\left(X^{\uparrow}, Y^{\downarrow}, I\right) \text { for some } C\right\},
\end{aligned}
$$

and analogously for $\operatorname{Ext}\left(X^{\cap}, Y^{\cup}, I\right), \operatorname{Int}\left(X^{\cap}, Y^{\cup}, I\right), \operatorname{Ext}\left(X^{\wedge}, Y^{\vee}, I\right)$, and $\operatorname{Int}\left(X^{\wedge}, Y^{\vee}, I\right)$.

A fundamental connection between the row and column spaces on one hand, and the concept lattices on the other hand, is described in the following theorem $\left(I^{T}\right.$ denotes the transpose of $\left.I\right)$.

Theorem 3. For a matrix $I \in L^{n \times m}, X=\{1, \ldots, n\}, Y=\{1, \ldots, m\}$, we have

$$
\begin{aligned}
& R_{i}(I)=\operatorname{Int}\left(X^{\cap}, Y^{\cup}, I\right)=\operatorname{Ext}\left(Y^{\wedge}, X^{\vee}, I^{T}\right), \\
& R_{c}(I)=\operatorname{Int}\left(X^{\uparrow}, Y^{\downarrow}, I\right)=\operatorname{Ext}\left(Y^{\uparrow}, X^{\downarrow}, I^{T}\right), \\
& C_{i}(I)=\operatorname{Ext}\left(X^{\wedge}, Y^{\vee}, I\right)=\operatorname{Int}\left(Y^{\cap}, X^{\cup}, I^{T}\right), \\
& C_{c}(I)=\operatorname{Ext}\left(X^{\uparrow}, Y^{\downarrow}, I\right)=\operatorname{Int}\left(Y^{\uparrow}, X^{\downarrow}, I^{T}\right) .
\end{aligned}
$$

Proof. $R_{i}(I)=\operatorname{Int}\left(X^{\cap}, Y^{\cup}, I\right)$ was established in [6]. The second equality of (10) is immediate. (12) is a consequence of (10) and when taking a transpose of $I$, extents and intents switch their roles.

Sketch of the proof of (11): The assertion follows from the fact that $D \in$ $\operatorname{Int}\left(X^{\uparrow}, Y^{\downarrow}, I\right)$ iff $D=C^{\uparrow}$ for some $C$, i.e. $D(j)=\bigwedge_{i \in X}\left(C(i) \rightarrow I_{i j}\right)$, which means that $D$ is the $\Lambda$-intersection of $\rightarrow$-shifts $C(i) \rightarrow I_{i_{-}}$of rows $I_{i_{-}}$of $I$. The set of all such intersections may be shown to form a c-space and it is the least c-space containing all rows of $I$.

The following theorem provides us with the relationships generalizing the well-known relationship between the row (column) space of a Boolean product of two Boolean matrices and the row (column) spaces of the two matrices.

Theorem 4. For $A \in L^{n \times k}$ and $B \in L^{k \times m}$,

$$
\begin{aligned}
& R_{i}(A \circ B) \subseteq R_{i}(B), \\
& C_{i}(A \circ B) \subseteq C_{i}(A), \\
& R_{c}(A \triangleleft B) \subseteq R_{c}(B), \\
& C_{c}(A \triangleright B) \subseteq C_{c}(A) .
\end{aligned}
$$

In addition,

$$
\begin{array}{r}
C_{c}(A \triangleleft B) \subseteq \operatorname{Ext}\left(X^{\cap},\{1, \ldots, k\}^{\cup}, A\right), \\
R_{c}(A \triangleright B) \subseteq \operatorname{Int}\left(\{1, \ldots, k\}^{\wedge}, Y^{\vee}, B\right) .
\end{array}
$$

Proof. The assertions follow from Theorem 3 and [6, Theorem 4] describing relationships of concept lattices of compositions of fuzzy relations; the full proof is omitted. 


\section{$3.2 \quad$ Green's Relations}

Green's relations [13] originated in semigroup theory and play an important role in Boolean matrix equations and other areas of Boolean matrix theory [16]. We now establish their basic properties for matrices with entries from residuated lattices. We take advantage of the fact that the $n \times n$ matrices with entries from a residuated lattice form semigroup with respect to $\circ$.

Definition 3. For $n \times n$ matrices $I$ and $J$, Green's relations $\mathcal{L}, \mathcal{R}$, and $\mathcal{D}$ are defined as follows:

- IL J iff $\left\{M \circ I \mid M \in L^{n \times n}\right\}=\left\{M \circ J \mid M \in L^{n \times n}\right\}$, i.e. I and J generate the same left ideal (we say that $I$ and $J$ are $\mathcal{L}$-equivalent).

- IR J iff $\left\{I \circ M \mid M \in L^{n \times n}\right\}=\left\{J \circ M \mid M \in L^{n \times n}\right\}$, i.e. I and $J$ generate the same right ideal (we say that $I$ and $J$ are $\mathcal{R}$-equivalent).

- IDJ iff there exists matrix $K \in L^{n \times n}$ such that $I \mathcal{L} K$ and $K \mathcal{R} J$.

Remark 4. (1) There exist several other ways to define Green's relations. For example:

- IR $J$ iff there exist $A, B$ such that $I \circ A=J$ and $J \circ B=I$.

- ID $J$ iff there exists $K$ such that $I \mathcal{R} K$ and $K \mathcal{L} J ; \mathcal{D}$ is the supremum of $\mathcal{L}$ and $\mathcal{R}$ in the lattice of equivalence relations.

(2) Due to lack of space, we omit the other two Green's relations, denoted usually $\mathcal{H}$ and $\mathcal{J}$.

Theorem 5. (1) IL $J$ iff $R_{i}(I)=R_{i}(J)$.

(2) IR $J$ iff $C_{i}(I)=C_{i}(J)$

Proof. (1) $\left\{M \circ I \mid M \in L^{n \times n}\right\}=\left\{M \circ J \mid M \in L^{n \times n}\right\}$ iff $\left\{M \circ I \mid M \in L^{1 \times n}\right\}=$ $\left\{M \circ J \mid M \in L^{1 \times n}\right\}$. Now, an immediate reflection shows that the latter condition is equivalent to $\operatorname{Int}\left(X^{\cap}, Y^{\cup}, I\right)=\operatorname{Int}\left(X^{\cap}, Y^{\cup}, J\right)$., i.e. by Theorem $3, I$ and $J$ have the same row i-spaces.

(2) The proof is similar to that of (1).

Theorem 6. Let $B$ be $a k \times m$ matrix and $I$ be an $n \times m$ matrix. $R_{i}(I) \subseteq R_{i}(B)$ iff there exists an $n \times k$ matrix $A$ such that $I=A \circ B$.

Proof. " $\Rightarrow$ ": Let $R_{i}(I) \subseteq R_{i}(B)$, i.e. $\operatorname{Int}\left(X^{\cap}, Y^{\cup}, I\right) \subseteq \operatorname{Int}\left(X^{\cap}, Y^{\cup}, B\right)$. Every $H \in \operatorname{Int}\left(X^{\cap}, Y^{\cup}, B\right)$ can be expressed as $\bigvee_{1<l<k} c_{l} \otimes B_{l_{-}}$. Thus every $H \in$ $\operatorname{Int}\left(X^{\cap}, Y^{\cup}, I\right)$ can be expressed as $\bigvee_{1 \leq l \leq k} c_{l} \otimes B_{l_{-}}$. Therefore, a row $I_{i_{-}}$of $I$ can be expressed as $\bigvee_{1 \leq l_{i} \leq k} c_{l_{j}} \otimes B_{l_{-}}$. We get the required matrix $A$ by putting $A_{i l}=c_{l_{j}}$.

"६": This was established in Theorem 4 .

In a similar way, we can establish other theorems describing the inclusion of the row and column spaces of $I$ in the corresponding spaces of $B$ (we omit them due to lack of space). 
Theorem 7. If I L $J$ and $A \circ B=I$ then there exists $A^{\prime}$ such that $A^{\prime} \circ B=J$.

Proof. Follows from (1) of Theorem 5 and Theorem 6.

An analogous theorem holds true for the equivalence relation $\mathcal{R}$. Using Theorem 7 and the analogous version for $\mathcal{R}$, we get the following corollary regarding the Schein rank of $\mathcal{D}$-equivalent matrices.

Theorem 8. If ID $J$ then $\rho_{s o}(I)=\rho_{s}(J)$.

In the following theorem, $\mathcal{B}\left(X^{\cap}, Y^{\cup}, I\right) \approx \mathcal{B}\left(X^{\cap}, Y^{\cup}, J\right)$ denotes that $\mathcal{B}\left(X^{\cap}, Y^{\cup}, I\right)$ and $\mathcal{B}\left(X^{\cap}, Y^{\cup}, J\right)$ are isomorphic lattices.

Theorem 9. ID $J$ iff $\mathcal{B}\left(X^{\cap}, Y^{\cup}, I\right) \approx \mathcal{B}\left(X^{\cap}, Y^{\cup}, J\right)$.

Proof. Sketch: " $\Rightarrow$ ": Let $I \mathcal{D} J$. There there exists $K$ such that $I \mathcal{L} K$ and $K \mathcal{R} J$. Hence, we have $\operatorname{Int}\left(X^{\cap}, Y^{\cup}, I\right)=\operatorname{Int}\left(X^{\cap}, Y^{\cup}, K\right)$ by Theorem 5. Let now $T$ and $S$ be matrices for which $K \circ S=J$ and $J \circ T=K$ (they exists according to the above remark). Consider the mappings

$$
\begin{aligned}
& \cap_{S}: \operatorname{Int}\left(X^{\cap}, Y^{\cup}, K\right) \rightarrow \operatorname{Int}\left(X^{\cap}, Y^{\cup}, J\right), \\
& \cap_{T}: \operatorname{Int}\left(X^{\cap}, Y^{\cup}, J\right) \rightarrow \operatorname{Int}\left(X^{\cap}, Y^{\cup}, K\right) .
\end{aligned}
$$

One can show that $\cap_{S} \cap_{T}$ and $\cap_{T} \cap_{S}$ are identities. Therefore, $\operatorname{Int}\left(X^{\cap}, Y^{\cup}, K\right)$ and $\operatorname{Int}\left(X^{\cap}, Y^{\cup}, J\right)$ are isomorphic, establishing that $\mathcal{B}\left(X^{\cap}, Y^{\cup}, I\right)$ and $\mathcal{B}\left(X^{\cap}, Y^{\cup}, J\right)$ are isomorphic lattices. The proof of " $\Leftarrow$ " omitted due to lack of space.

The above notions and results in this section have their counterparts for $\triangleleft$ and $\triangleright$-compositions of matrices. In the rest of this section, we present some of them without proofs.

Definition 4. For $n \times n$ matrices $I$ and $J$, Green's relations $\mathcal{L}_{\triangleleft}, \mathcal{R}_{\triangleright}$, and $\mathcal{D}_{\triangleleft \triangleright}$ are defined as follows:

- I $\mathcal{L}_{\triangleleft} J$ iff $\left\{M \triangleleft I \mid M \in L^{n \times n}\right\}=\left\{M \triangleleft J \mid M \in L^{n \times n}\right\}$.

- IR $\mathcal{R}_{\triangleright} J$ iff $\left\{I \triangleright M \mid M \in L^{n \times n}\right\}=\left\{J \triangleright M \mid M \in L^{n \times n}\right\}$.

- ID $\mathcal{D}_{\triangleleft \triangleright} J$ iff there exists matrix $K \in L^{n \times n}$ such that $I \mathcal{L}_{\triangleleft} K$ and $K \mathcal{R}_{\triangleright} J$.

Theorem 10. (1) I $\mathcal{L}_{\triangleleft} J$ iff $R_{c}(I)=R_{c}(J)$.

(2) $I \mathcal{R}_{\triangleright} J$ iff $C_{c}(I)=C_{c}(J)$

Theorem 11. Let $B$ be a $k \times m$ matrix and $I$ be an $n \times m$ matrix. $R_{c}(I) \subseteq R_{c}(B)$ iff there exists an $n \times k$ matrix $A$ such that $I=A \triangleleft B$.

Theorem 12. If I $\mathcal{L}_{\triangleleft} J$ and $A \triangleleft B=I$ then there exists $A^{\prime}$ such that $A^{\prime} \triangleleft B=J$.

Theorem 13. If ID $\mathcal{D}_{\triangleleft \triangleright} J$ then $\rho_{s \triangleleft}(I)=\rho_{s \triangleleft}(J)$. 


\section{Conclusions}

We established properties of structures associated with matrices with entries from residuated lattices. By doing so, we generalized the properties known from Boolean matrix theory and provided links to structures known from formal concept analysis. The line of research started in this paper and its predecessor is to be continued to establish foundations for a calculus of matrices with entries from residuated lattices. The usefulness of such calculus for data analysis is demonstrated in [8].

\section{Acknowledgment}

Supported by grant No. 202/10/0262 of the Czech Science Foundation and by research plan MSM 6198959214.

\section{References}

1. Belohlavek R.: Fuzzy Galois connections. Math. Logic Quarterly 45,4 (1999), 497504.

2. Belohlavek R.: Fuzzy closure operators. J. Math. Anal. Appl. 262(2001), 473-489.

3. Belohlavek R.: Fuzzy Relational Systems: Foundations and Principles. Kluwer, Academic/Plenum Publishers, New York, 2002.

4. Belohlavek R.: Concept lattices and order in fuzzy logic. Annals of Pure and Applied Logic 128(1-3)(2004), 277-298.

5. Belohlavek R., Funioková T.: Fuzzy interior operators. Int. J. General Systems 33(4)(2004), 315-330.

6. Belohlavek R., Konecny J.: Operators and spaces associated to matrices with grades and their decompositions. Proc. NAFIPS 2008, 2008, 6 pages; IEEE Press.

7. Belohlavek R.: Optimal triangular decompositions of matrices with entries from residuated lattices. Int. J. of Approximate Reasoning 50(8)(2009), 1250-1258.

8. Belohlavek R., Vychodil V.: Factor analysis of incidence data via novel decomposition of matrices. Lecture Notes in Artificial Intelligence 5548(2009), 83-97.

9. Belohlavek R., Vychodil V.: Discovery of optimal factors in binary data via novel method of matrix decomposition. J. Computer and System Sciences 76(1)(2010), $3-20$.

10. Belohlavek R.: Optimal decompositions of matrices with entries from residuated lattices. Conditionally accepted to J. Logic and Computation.

11. Ganter B., Wille R.: Formal Concept Analysis. Mathematical Foundations. Springer, Berlin, 1999.

12. Georgescu G., Popescu A.: Non-dual fuzzy connections. Archive for Mathematical Logic 43(2004), 1009-1039.

13. Green J. A.: On the structure of semigroups. Annals of Mathematics 54(1)(1951), $163-172$.

14. Hájek P.: Metamathematics of Fuzzy Logic. Kluwer, Dordrecht, 1998.

15. Harman H. H.: Modern Factor Analysis, 2nd Ed. The Univ. Chicago Press, Chicago, 1970.

16. Kim K. H.: Boolean Matrix Theory and Applications. M. Dekker, 1982. 
17. Kohout L. J., Bandler W.: Relational-product architectures for information processing. Information Sciences 37(1-3): 25-37, 1985.

18. Pollandt S.: Fuzzy Begriffe. Springer-Verlag, Berlin/Heidelberg, 1997.

19. M. Ward, R. P. Dilworth. Residuated lattices. Trans. Amer. Math. Soc. 45 (1939), $335-354$. 